УДК $621.311+004.45$

\title{
СПОСОБЫ ПЕРЕДАЧИ ИНФОРМАЦИИ ОТ ВОЛЬТДОБАВОЧНЫХ ТРАНСФОРМАТОРОВ 6 -20 КВ В SCADA СИСТЕМУ ПО БЕСПРОВОДНЫМ И ПРОВОДНЫМ КАНАЛАМ СВЯЗИ
}

\author{
Андрианова Людмила Прокопьевна \\ д-р техн. наук, профессор \\ Рябишина Лилия Амировна \\ канд. техн. наук, доцент \\ Хуртов Владислав Евгеньевич
}

Уфимский государственный нефтяной технический университет

Аннотация: B статье представлен анализ типовых решений по организации передачи информации с вольтдобавочных трансформаторов (ВДТ), устанавливаемых в линиях электропередач 6-20 кВ, по беспроводному и проводному и каналам связи в систему сбора данных и оперативного контроля (SCADA) в диспетчерском управлении. Приведен перечень сигналов по телеизмерению (ТИ), телесигнализации (ТС) и телеуправлению (ТУ). Приведены структурные схемы передачи данных от ВДТ в диспетчерский пункт по каналу GPRS, и по проводным каналам, а также принципиальные схемы шкафа телемеханики ВДТ с беспроводной и проводной связью.

Ключевые слова: вольтдобавочные трансформаторы, интеграция в систему SCADA; перечень передаваемой информации; беспроводные и проводные линии связи; коммуникационное оборудование; шкаф телемеханики.

\section{METHODS OF TRANSMITTING INFORMATION FROM 6-20 KV VOLT-BOOST TRANSFORMERS TO THE SCADA SYSTEM VIA WIRELESS AND WIRED COMMUNICATION CHANNELS}

\author{
Andrianova Lyudmila Prokopyevna \\ Ryabishina Lilia Amirovna \\ Hurtov Vladislav Evgenievich
}

\begin{abstract}
The article considers typical solutions for the organization of information transmission from voltage-add-on transformers (VDT) installed in power
\end{abstract}


lines of $6-20 \mathrm{kV}$, via wireless and wired communication channels to the data collection and operational control system (SCADA)in the dispatcher control. The list of signals for tele-measurement (TI), tele-signalization (TS) and remote control (TU) is given. Block diagrams of data transmission from the VDT to the control room via the GPRS channel, and via wired channels, as well as schematic diagrams of the VDT telemechanics cabinet with wireless and wired communication are presented.

Key words: voltage-add-on transformers, integration into the SCADA system; list of transmitted information; wireless and wired communication lines; communication equipment; telemechanics cabinet.

Современные вольтодобавочные трансформаторы ВДТ 6-20 кВ, устанавливаемые в линиях электропередачи в качестве автоматических регуляторов напряжения, являются компонентами цифровой электрической сети $[1,2,4,7]$. В соответствии с СТО 34.01-21-005-2019 ПАО «Россети» [7] цифровая электрическая сеть должна соответствовать таким критериям, как дистанционная наблюдаемость параметров и режима работы подстанций, распределительных пунктов (РП), трансформаторных подстанций (ТП), линий электропередач (ЛЭП) со всеми элементами сетей и интеграция всех элементов сети в SCADA систему с возможностью телеуправления.

Интеграция пунктов регулирования напряжения (ПРН) с ВДТ 6-20 кВ в SCADA систему возможна при использовании каналов связи и протоколов передачи данных, представленных в таблице 1 [3, с. 37].

Таблица 1

Каналы и протоколы передачи данных в SCADA систему

\begin{tabular}{|c|c|c|}
\hline $\begin{array}{c}\text { Тип дистанционного } \\
\text { управления }\end{array}$ & $\begin{array}{c}\text { Канал передачи данных } \\
\text { SCADA }\end{array}$ & Протокол передачи данных \\
\hline \multirow{3}{*}{ SCADA } & GRPS/GSM & МЭК 60870-5-104 \\
\cline { 2 - 3 } & ВОЛC, RS232/485 & МЭК 61850 90-2. \\
& \multicolumn{2}{|c}{ при необходимости МЭК } \\
& $60870-5-104$ \\
\hline
\end{tabular}

Перечень передаваемой информации с ВДТ 6-20 кВ представлен в таблицах 2-4 [3, с. 41]. 
Таблица 2

Перечень сигналов по телеизмерению (ТИ)

\begin{tabular}{|c|c|c|}
\hline $\begin{array}{l}\text { № } \\
\Pi / \Pi\end{array}$ & Наименование & Единица измерения \\
\hline \multicolumn{3}{|c|}{ Измерение электрических параметров } \\
\hline 1 & Напряжение со стороны источника & кB \\
\hline 2 & Напряжение со стороны нагрузки & кB \\
\hline 3 & Ток в линии & $\mathrm{A}$ \\
\hline 4 & Коэффициент мощности & o.e. \\
\hline \multicolumn{3}{|c|}{ Интегральные параметры ТИ } \\
\hline 5 & Количество переключений & \\
\hline \multicolumn{3}{|c|}{ Уставки по прямому и по обратному направлениям мощности } \\
\hline 6 & Уставка по напряжению & $\mathrm{B}$ \\
\hline 7 & Уставка по диапазону чувствительности & $\mathrm{B}$ \\
\hline 8 & Уставка по времени & $\mathrm{c}$ \\
\hline 9 & $\begin{array}{l}\text { Уставка по компенсации падения напряжения } \\
\text { на активном сопротивлении }\end{array}$ & B \\
\hline 10 & $\begin{array}{l}\text { Уставка по компенсации падения напряжения } \\
\text { на реактивном сопротивлении }\end{array}$ & B \\
\hline
\end{tabular}

Таблица 3

\section{Перечень сигналов по телесигнализации (ТС)}

\begin{tabular}{|l|l|l|}
\hline 1 & Режим управления & Автоматическое / Ручное \\
\hline 2 & Телеуправление & Включено / Отключено \\
\hline 3 & Направление потока мощности & Прямое / Обратное \\
\hline 4 & $\begin{array}{l}\text { Нейтральное положение переключателя РПН } \\
\text { (регулирование напряжения под нагрузкой) }\end{array}$ & Да / Нет \\
\hline 5 & Неисправность ВДТ & Да / Нет \\
\hline
\end{tabular}

Таблица 4

Перечень сигналов по телеуправлению (ТУ)

\begin{tabular}{|c|l|c|}
\hline $\begin{array}{c}\text { № } \\
\text { п/п }\end{array}$ & \multicolumn{1}{|c|}{ Наименование } & Единица измерения \\
\hline \multicolumn{2}{|c|}{ Измерение электрических параметров } \\
\hline 1 & Переключение ступени & на повышение \\
\hline 2 & Переключение ступени & на понижение \\
\hline 3 & Переключение режима & Автоматический / Ручной \\
\hline \multicolumn{2}{|c|}{ Уставки по прямому и по обратному направлениям мощности } \\
\hline 6 & Уставка по напряжению & $\mathrm{B}$ \\
\hline 7 & Уставка по диапазону чувствительности & $\mathrm{B}$ \\
\hline 8 & Уставка по времени & $\mathrm{c}$ \\
\hline
\end{tabular}




\begin{tabular}{|c|l|c|}
\hline 9 & $\begin{array}{l}\text { Уставка по компенсации падения напряжения } \\
\text { на активном сопротивлении }\end{array}$ & $\mathrm{B}$ \\
\hline \multirow{2}{*}{10} & $\begin{array}{l}\text { Уставка по компенсации падения напряжения } \\
\text { на реактивном сопротивлении }\end{array}$ & $\mathrm{B}$ \\
\hline
\end{tabular}

Организация передачи данных по каналу беспроводной связи (GPRS /GSM). Коммуникационное оборудование ПРН с ВДТ 6-20 кВ (нижний уровень) включает контроллер сбора информации и конвертации протокола, преобразователь интерфейса, блок питания с выходным напряжением 12 В постоянного тока, а также автомат защиты. Коммуникационное оборудование ПРН устанавливаются в шкафу телемеханики. Шкаф телемеханики выполняется в виде отдельного металлического шкафа со степенью защиты IP не ниже 43 и закрепляется на баке ВДТ (рис. 1) или на опоре в непосредственной близости от ВДТ.

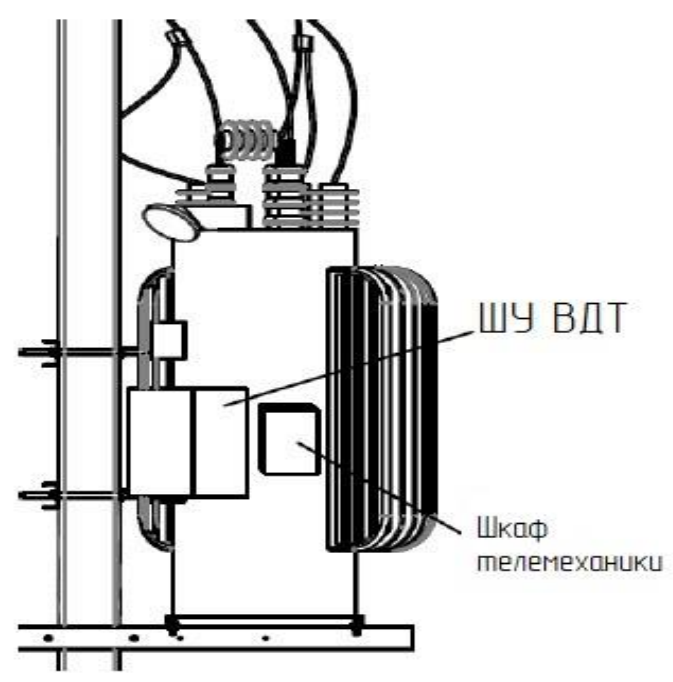

Рис. 1. Крепления шкафа телемеханики на баке ВДТ: ШУ ВДТ - шкаф управления

Питание шкафа телемеханики производится от шкафа управления ШУ ВДТ посредством ввода кабелей через гермовводы.

Принципиальная схема шкафа телемеханики ВДТ представлена на рисунке 2. 




Рис. 2. Принципиальная схема шкафа телемеханики с беспроводной связью: SF - Выключатель автоматический ВА 47-23 2p 1с; A1 - Контроллер сбора информации и конвертации протокола; A2 - Роутер GPRS/GSM; URG - Блок питания AC-DC 12 V; WD - Антенна 905 5dB SMA

Структурная схема передачи данных от ВДТ представлена на рисунке 3. Структурная схема передачи данных на верхний уровень - диспетчерский пункт представлена на рисунке 4.

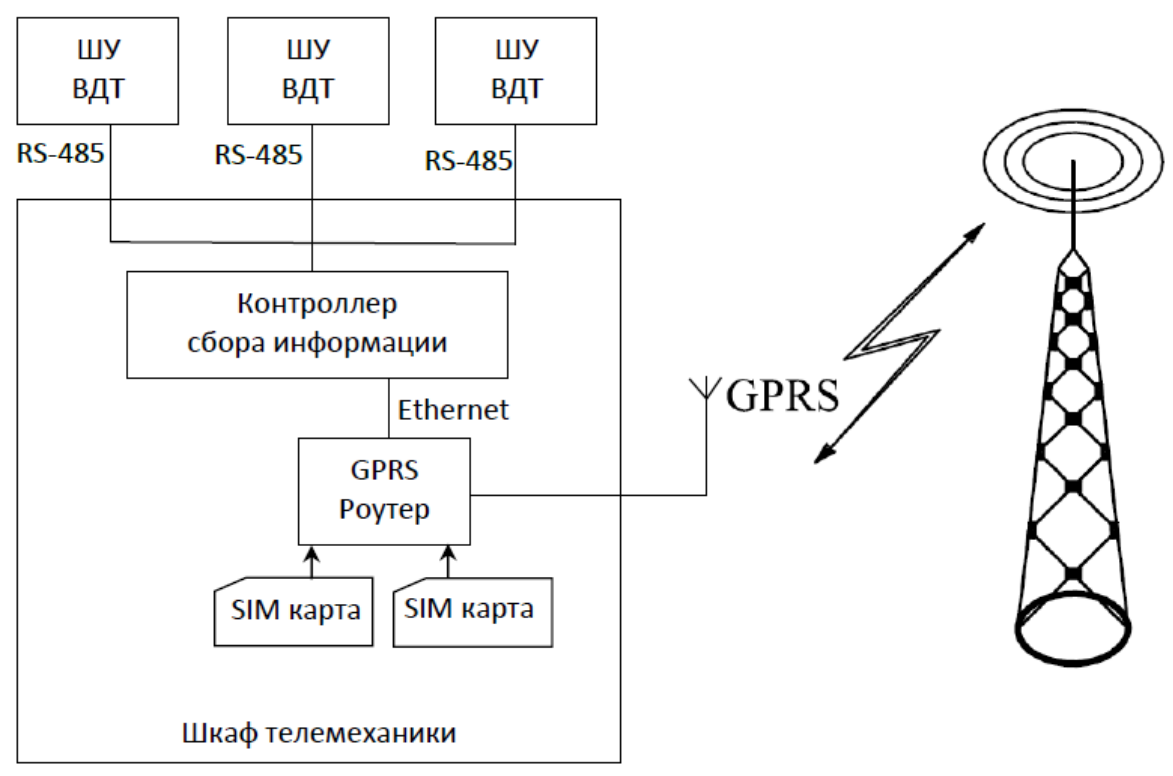

Рис. 3. Структурная схема передачи данных от ВДТ 
Диспетчерский пункт

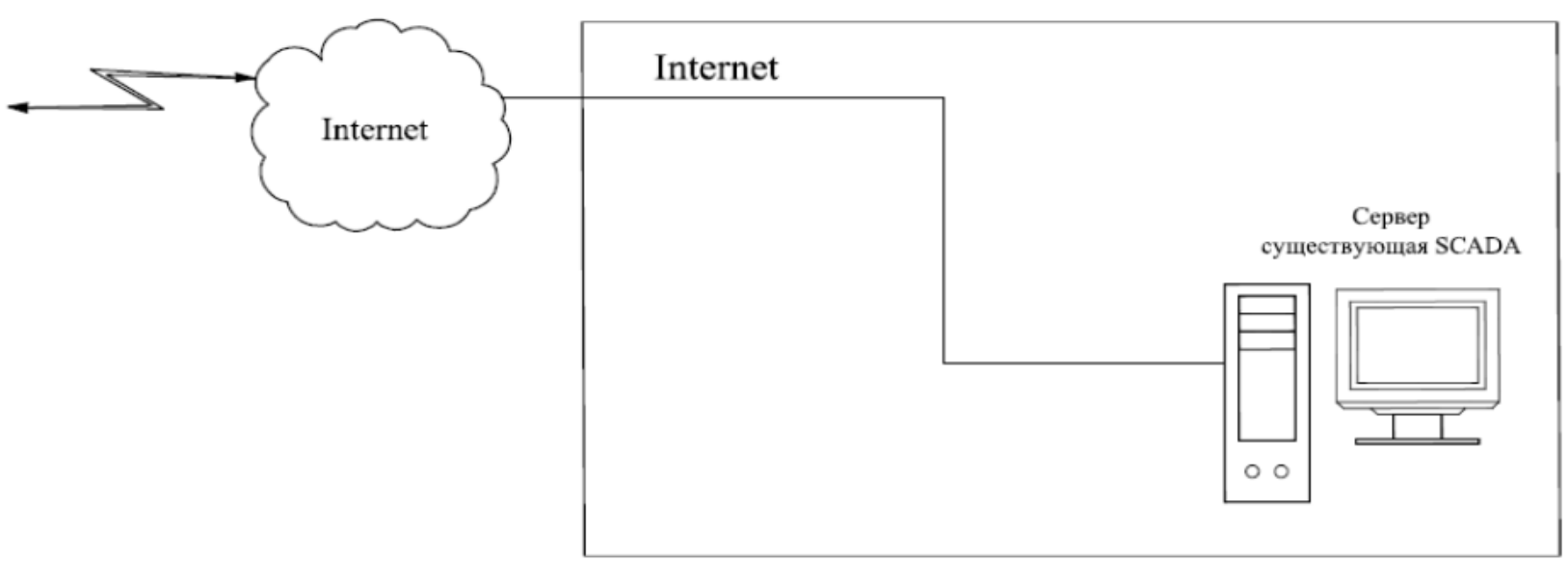

Рис. 4. Структурная схема передачи данных в диспетчерский пункт

При использовании беспроводного канала связи GRPS/GSM в случае слабого или неустойчивого сигнала сотовой сети в месте установки ВДТ необходимо применять направленную антенну или использовать две сим-карты разных сотовых операторов [3, с. 37].

Передача данных по проводным линиям связи. При передаче данных по протоколу МЭК 60870-101/104 и Modbus преобразователь интерфейса подключается к конвертору протокола, при передаче данных по протоколу DNP3.0 преобразователь интерфейсов подключается напрямую к ШУ ВДТ.

Коммуникационное оборудование нижнего уровня (ПРН с ВДТ 6-20 кВ) устанавливаются в отдельном металлическом шкафу со степенью защиты IP не ниже 43. Шкаф телемеханики крепится на баке ВДТ или на опоре в непосредственной близости от ВДТ. Марка преобразователя зависит от типа выбранного интерфейса. Питание шкафа телемеханики производится от ШУ ВДТ. Для обеспечения герметичности шкафа, ввод антенн и цепи вторичной коммутации и кабеля питания выполняются через гермовводы.

Принципиальная схема шкафа телемеханики с проводной связью представлена на рисунке 5. 




Рис. 5. Принципиальная схема шкафа телемеханики с проводной связью: SF - Выключатель автоматический ВА 47-29 2p 1с; A1 - Контроллер сбора информации и конвертации протокола; А2 - Преобразователь (зависит от интерфейса передачи данных); URG - Блок питания AC-DC 12 V

Тип устанавливаемого преобразователя А2 и линии связи зависит от типа интерфейса передачи данных, и может быть: ВОЛС, RS-485, Ethernet, Радио-Ethernet. В диспетчерском пункте устанавливается преобразователь интерфейсов для соединения с сервером SCADA по интерфейсу Ethernet. Структурная схема передачи данных представлена на рисунке 6. 




Рис. 6. Структурная схема передачи данных от шкафа управления ВДТ с проводной линией связи (**зависит от интерфейса)

При эксплуатации шкафов телемеханики как с беспроводной, так и с проводной связью в районах с холодным климатом предусматривается автоматический обогрев.

Заключение. Изложенные принципы интеграции автоматических пунктов регулирования напряжения с ВДТ 6-20 кB в SCADA системы способствуют повышению знаний обучающихся по направлению Электроэнергетика и электротехника, и могут быть полезны специалистам электроэнергетической отрасли в рамках повышения квалификации.

\section{Список литературы}

1. Вольтодобавочные трансформаторы. Общие технические требования : СТО 34.01-3.2-013-2017. - ПАО «Россети»; введ. 02.08.2017. - 43 с.

2. Методические указания по применению вольтодобавочных трансформаторов (пунктов регулирования напряжения) 6-20 кB и вольтодобавочных трансформаторов 0,4 кВ в линиях электропередачи распределительных сетей. Том 1.1. Методические указания : СТО 34.01-3.2.17014.1-2020. - ПАО «Россети»; введ. 04.03.2020. - 44с.

3. Методические указания по применению вольтодобавочных трансформаторов (пунктов регулирования напряжения) 6-20 КВ и 
вольтодобавочных трансформаторов 0,4 КВ в линиях электропередачи распределительных сетей. Том 1.2. Типовые решения : СТО 34.01-3.2.17-014.22020. - ПАО «Россети»; введ. 04.03.2020. - 44c.

4. Распределительные электрические сети напряжением 0,4-110 кВ. Требования к технологическому проектированию: СТО 34.01-21.1-001-2017. ПАО «Россети»; введ. 02.08.2017. - 238 с.

5. Регламент управления качеством электрической энергии в распределительных электрических сетях дочерних зависимых обществ : СТО 34.01-39.3-003-2018. - ПАО «Россети»; введ. 03.05. 2018. - 23с.

6. Системы диспетчерского управления в электроэнергетике. Система сбора данных и оперативного контроля (SCADA) в диспетчерском управлении : СТО 59012820.35.240.50.004-2011; введ. 24.06.2011. - Москва, 2011. - 53 с.

7. Цифровая электрическая сеть. Требования к проектированию цифровых распределительных электрических сетей 0,4-220 кВ : СТО 34.01-21005-2019. ПАО «Россети»; введ. 29.03.2019.; акт. 01.01.2021. - Москва, 2019. $96 \mathrm{c}$.

() Андрианова Л.П., Рябишина Л.А., Хуртов В.Е. 